\title{
Electrically charged droplet: case study of a simple generator
}

\author{
Martin Brandenbourger and Stéphane Dorbolo
}

\begin{abstract}
We studied the behavior of a simple apparatus that allows to charge droplets by induction. We imposed the following constraints (1) any liquid should be charged (mainly whatever its surface tension) and (2) the system is to remain as simple as possible. The design of the device results of a compromise regarding both constraints. The device developed is based on a planar capacitor in which the droplet are generated. The influence of the nature of the liquid (i.e. presence of ions in solution, polarity, surface tension and conductivity) on the charge induced was measured and was found to be independent of the liquid properties (except for the silicone oil). We deduced that, in the considered configuration, fluids behave like a "perfect conductor" as soon as conductive relaxation time is smaller than the droplet ejection time. Under this condition, the charge has the time to move in the droplet to reach the electrostatic regime.
\end{abstract}

\section{Introduction}

Basically, rain falling from thunderclouds is composed of electrically charged droplets [1]. Since Rayleigh's [?] experiments the electrically charged droplet were intensively studied regarding applications. Indeed, they are the basis of many applications such as electrospray [3], electrowetting [4], or electrospinning [5]. Electrically charged droplets have also been studied from a fundamental point of view. Indeed, electrically charged droplets lead to impressive behaviors such as the Coulomb explosion [2] or particular interactions between droplets [6]. Moreover, the understanding of the charge of a droplet is linked to the study of charging cloud droplets in thunderstorms.

Three different ways to charge droplets can be found in literature : triboelectricity, radioactive radiations or charge by influence. The first two manners are frequently used in Millikan experiments [7]. They have the advantage to charge droplets easily, without any "complicated" set-up. However, both techniques do not allow to predict the charge induced in the droplet. On the contrary, the charge by influence allows to determine precisely the charge in the droplet. Indeed, as we will show, the geometry of the set-up (and the voltage applied to it) corresponds to a given amount of charges induced in the droplet. However, the mechanism of charging by induction a liquid is more complex to explain than the charge by induction in a solid conductor.

Charged droplets generators using the charge by influence phenomenon have already been studied in the past years. For example, Jones and Thong [8] have proposed a geometry for a charged droplet generator using charge by induction. Their geometry, composed of one needle and one plate, is commonly used in electrospray devices [9]. In the context of this kind of device, they proposed also a theoretical approach to predict the charge induced in the droplet. Assuming that the generator and

Received . Revision received . Accepted . Revision accepted .

M. Brandenbourger.

GRASP, Physics Department, University of Liège, B-4000 Liège, Belgium.

S. Dorbolo.

F.R.S.-FNRS, GRASP, Physics Department, University of Liège, B-4000 Liège, Belgium. the liquid are perfect conductors, it is possible to calculate the electric field induced in the charged droplet generator. Knowing the electric field in the droplet, they calculate the charge density in the droplet and thus the total charge in the droplet.

Electrically speaking, a droplet can be modeled by a resistor in parallel with a capacity. Consequently, a characteristic time $\tau$ can be defined. When an electrical phenomenon occurs during a time below $\tau$, capacitive effects have to be taken into account. On the other hand, for duration larger than $\tau$, the system behaves like a resistor. The characteristic time is given by

$$
\tau=\epsilon_{r} \epsilon_{0} / \sigma
$$

where $\epsilon_{r}$ and $\sigma$ are the relative permittivity and the conductivity of the liquid, $\epsilon_{0}$ being the vacuum permittivity. When the typical time $\tau_{d}$ to generated one charged droplet is much larger than $\tau$, the droplet can be qualified as a "perfect conductor", i.e. the capacitive effects cannot be observed [10].

In this paper, we explain how we developed an apparatus which permits to charge droplets by influence. For our device, we required that (i) the design is as simple as possible and (ii) the system is to be able to charge any liquid regardless its surface tension. To reach these goals, the charged droplet dispenser was designed such as an homogeneous electric field is generated. For this, we propose a geometry which looks like a planar capacitor. Indeed, theoretically, this kind of configuration can be described by a simple law. It is possible to calculate the charge induced in the droplet with the same arguments as Jones et al [8]. Namely, the charge by unit area on a planar capacitor is :

[1] $\quad \frac{Q}{S}=\frac{V \epsilon_{0}}{d}$

where $Q$ is the charge of the droplet, $S$ the surface of the droplet, $V$ the tension applied on the capacitor and $d$ is the distance between the two parts of the capacitor. Under "perfect conductor" conditions, the total charge induced in the droplet is given by

$$
Q=\frac{V \epsilon_{0} 4 \pi r_{d}^{2}}{d}
$$


where $r_{d}$ is the droplet radius. This later equation is a simple relation which allows to easily predict the charge induced in the droplet.

Unfortunately, in practice, a charged droplet cannot be created from a planar capacitor. Indeed, a droplet formed on the top plate wets the surface before falling from the top plate and the bottom plate needs a hole in its center to allow the droplet to fall away from the generator. That is why the device developed in this study is composed of a needle which pierces the top plate and a hole in the bottom plate.

After a description of the experimental set-up, we present the study of the different geometrical parameters that may influence the charge induced in the droplet. Afterwards, we tested several liquids to check the robustness and the performance of our charged droplet dispenser. In order to cover a large variety of fluids, we chose to compare several properties like surface tension, polarity and ionicity of the fluid. Note also that the viscosity does not vary too much compared to the viscosity of the water. We found that most of the considered fluid behaves like a perfect conductor. Finally, the conclusion are drawn.

\section{Experimental set up}

\subsection{Charged droplet generator}

The charged droplet generator is based on the charge by induction mechanism. This kind of device generates an electric field which attracts positive (or negative) charges in a pendant droplet or/and extracts negative (or positive) charges out of a pendant droplet (see, for example [8]).

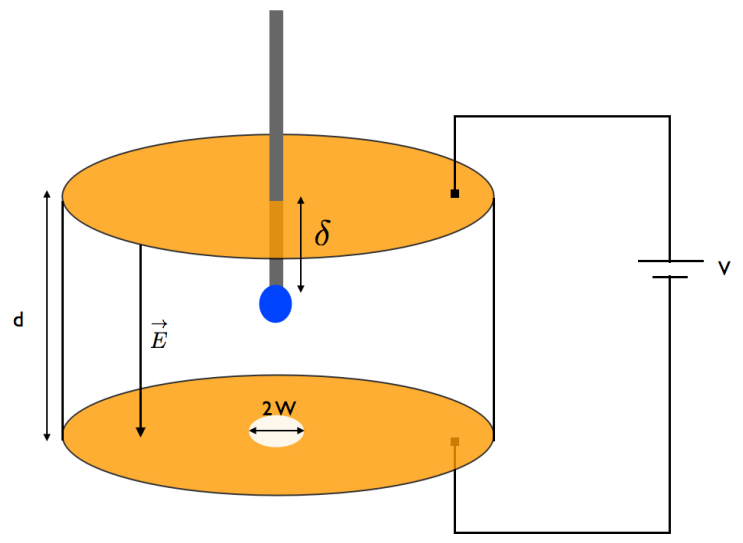

Fig. 1. (Color online) Sketch of the charged droplet generator. Both metal disks are represented by yellow circles. They are separated by a polycarbonate ring (not represented). The needle is represented by the grey vertical line. The voltage between both plates allows to generate an electric field $(\vec{E})$ and then, to charge by influence the pendant droplet. Note that $\delta, d$ and $W$ are parameters to optimize.

The charged droplet dispenser is to be used for charging various liquids, which implies (i) to be able to generate high electric fields (ii) to be able to deal with low surface tension fluids. In order to tackle these constraints, the charged droplet generator was made of two metal disks, parallel and positioned along a vertical axis (see Fig. 1). The top plate was pierced by a needle from which droplets was formed. A hole was drilled in the center of the bottom plate to allow the droplet to fall away from the charged droplet generator.

Three parameters govern the intensity and the geometry of the electrical field between the plates: the piercing distance $\delta$ of the needle into the top plate, the radius $W$ of the hole in the bottom plate and the distance $d$ between both plates.

From a qualitative point of view, decreasing $d$ allows to increase the electrical field. However, if $d$ is too small, even small droplet may be larger than $d$ going out of the capacitor. Moreover, if $d$ is too small, electrical breakdown may occur. As for the hole pierced in the bottom plate, it should be as small as possible in order to induce large amount of charge in the droplet. However, the droplet is to be allowed to fall out of the condenser. Finally, the distance $\delta$ should be small to be as close as possible to a planar capacitor configuration. But, if it is too small, the droplet may stick to the top plate by capillarity effect. Regarding the different effects, compromises have to be found in order to reach our goal, see Sect.3.4.

The droplet radius is also an important parameter to determine as we are interested in the surface charge density, i.e. $\sigma=Q / 4 \pi r_{d}^{2}$. When a given voltage is applied, electrodes generate a vertical electric field which induces a given charge in the pendant droplet attached to the needle. Basically, the droplet faces three forces: its weight, the electrical attraction by the bottom electrode and the surface tension force due to the contact with the needle. The detachment occurs when the weight and the electrical attraction surpass the surface tension force. That relation reads

$$
\frac{4}{3} \pi r_{d}^{3} \rho g+\frac{1}{4 \pi \epsilon_{0}} \frac{\left(4 \pi r_{d}^{2} \sigma\right)^{2}}{\left(d-\delta-r_{d}\right)^{2}}>2 \pi r \gamma
$$

where $\rho$ is the density of the liquid, $g$ is the gravity, $\sigma$ is the charge surface density, $r$ is the radius of the needle and $\gamma$ is the surface tension. Taking typical values, namely $r_{d}=1 \mathrm{~mm}$, $\rho=1000 \mathrm{~kg} / \mathrm{m}^{3}, \sigma=10 \mathrm{nC} / \mathrm{m}^{2}$, we find that the electrostatic term can be neglected with respect to the weight. Consequently, the droplet detaches when the weight of droplet is larger than the capillary force. Note finally that the surface tension does depend on the surface charge [11]. However, we focus on low induction voltage (up to $200 \mathrm{~V}$ ). Consequently, the dependance of the surface tension on the charge can be neglected. To summarize, in the described conditions, the droplet size only depends on the liquid nature. The radii were directly determined by measuring the volume accumulated by 100 droplets.

Finally, the "perfect conductor" conditions, used to find the Eq. (2) have to be checked regarding the time to generate a droplet using our dispenser. The droplet were generated by manually actioning the piston of the syringe until the droplet detaches. The typical time for creating a droplet is 3 seconds while the detachment takes typically milliseconds. Consequently, the detachment time $\tau_{d}$ defined as the time required for the creation and the detachment of the droplet is of the order of several seconds. The conductive relation time $\tau$ has to be much smaller than some seconds to allow to consider the fluid as a perfect conductor. 


\subsection{Charge detection set-up : The Faraday cup}

The measurement of the charge of the droplet was performed by using a Faraday cup. This kind of device is usually used in ions beam experiments. Contrary to this scientific field, the Faraday cup developed here aims to measure the charge of mesoscopic objects, i.e. charged droplets. The Faraday cup created to achieve this goal is schematized in Fig. 2. The same kind of device was used in a recent work [12]. It was composed of two concentric cups. The air space between both cups was covered by an insulated cap to protect the inside of the device from charged droplet which could fall into the device. The inside cup was connected to the input "high" connector of an electrometer (Keithley 610C) while the outside cup was connected to the input "low" connector and to the chassis ground. The electrometer was set in coulombmeter mode. When a droplet falls into the Faraday cup, it discharges on the inside of the cup. Droplets were added in the cup up to reach the maximum measurable value of the electrometer. As the number of droplets is known, the charge per droplet can be accurately determined.

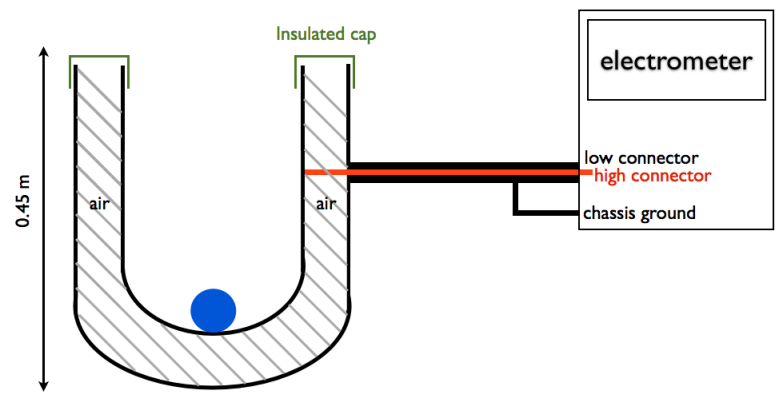

Fig. 2. (Color online) Illustration of the Faraday cup. The droplet falls into the inner cup and discharges. The electrometer measures the charge of the droplet in comparison to the chassis ground.

\section{Generator configuration}

This section aims to study the influence of the geometrical parameters $(d, W, \delta)$ on the charge induced in the droplet. All experiments performed for this purpose was made with bidistilled water.

\subsection{Influence of $d$}

First of all, the influence of $d$, the distance between both disks, is considered. Fig. 3 presents the charge measured on the droplet as a function of $d$ while $W=5 \mathrm{~mm}$ and $\delta=3$ $\mathrm{mm}$. The red curve is the fit made on the data between $d=5$ $\mathrm{mm}$ and $d=26 \mathrm{~mm}$ by the function

$$
\text { [4] } Q=\frac{\alpha}{d}
$$

where $\alpha$ is a fitting parameter. $\alpha$ is find to be equal to 0.23 $\mathrm{m} / \mathrm{pC}$. However, the calculated parameter by the $\mathrm{Eq}(2)$ is equal to $0.058 \mathrm{~m} / \mathrm{pC}$. The quantitative discrepancy between the $\alpha$ parameters comes from the hole $W$ and the needle $\delta$ that deviates the generator from the planar capacitor. However, the dependence in $1 / d$ is well found, which confirm the planar capacitor behavior of our device.

The largest deviation from the $1 / d$ law is obtained at $d=3$ $\mathrm{mm}$. Consequently, it seems that the law is in great agreement with the measurements except for small distance $d$. This last behavior can be explained by the study of $\delta$ and $W$ as we will see in the next section.

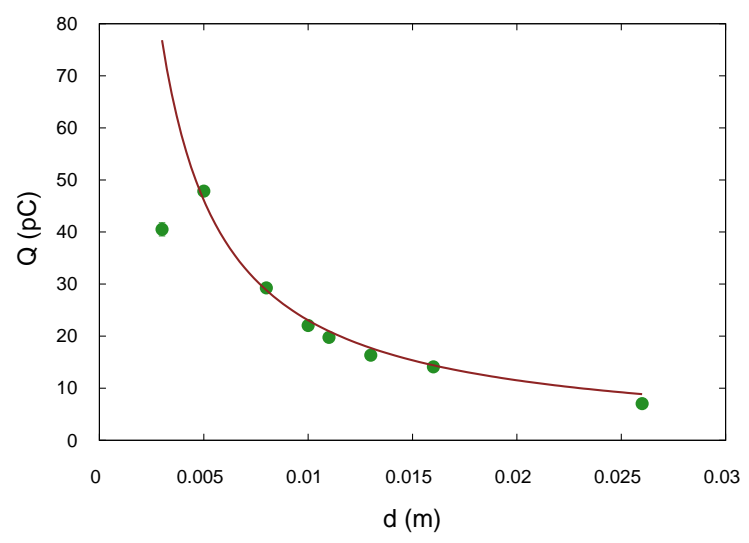

Fig. 3. (Color online) Charge induced in the droplet as a function of the distance between both plates $d(W=5 \mathrm{~mm}$ and $\delta=3$ $\mathrm{mm}$ ). Error bars are hidden by the points size. They correspond to a standard deviation calculated on 10 measurements. The red curve is a fit by the Eq. (4) between $d=5 \mathrm{~mm}$ and $d=26 \mathrm{~mm}$.

\section{2. influence of $\delta$ and $W$}

The effect of $\delta$ is directly linked to the distance $d$. Indeed, $\delta$ begins to be non-negligible as soon as it is comparable to $d$. Practically, the geometry of our charged droplet generator does not allow to vary continuously the distance $\delta$. Indeed, the needle has to be weld to the top disks to allow a good electrical connection. During the measurements on the effect of $d, \delta$ was fixed to $3 \mathrm{~mm}$. The study of the charge induced as a function of $d$ indicates that the measurements differs from the behavior described by the Eq. (2) when $d<5 \mathrm{~mm}$. As a consequence, the assumption that supposes that $\delta$ begins to be non-negligible when it is comparable to $d$ is confirmed.

We now consider the case of small distance $d$ (namely $d<5$ $\mathrm{mm}$ ) between the metal disks. In this case the presence of the needle (i.e $\delta$ ) becomes significant. Consequently, the geometry becomes different from the case of a planar capacitor. Indeed, the system approach the geometry described by Jones et al [8] (only a needle and a plate) and for very small $d$, the droplet begins to be out of the charged droplet generator before it detaches from the needle (which implies that the electric field does not apply on the whole droplet). Moreover, for short distances between circular plates, the hole in the bottom plate is not anymore negligible. As a consequence, the electric field generated by the system is smaller than expected. These arguments are confirmed by the measurement at very small $d$ (see 
Fig. 3) and by the study on the effect of the variation of $W$ (see further).

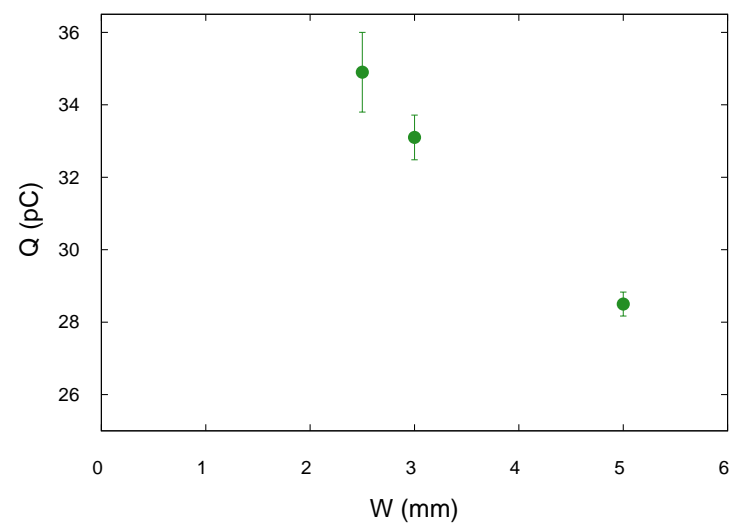

Fig. 4. (Color online) Charge induced in the droplet as a function of the radius of the hole in the bottom plate, $W(d=10 \mathrm{~mm}$ and $\delta=5 \mathrm{~mm}$ ). Error bars correspond to a standard deviation calculated on 10 measurements.

The effect of $W$ is shown in Fig. 4. Measurements was made for $d=10 \mathrm{~mm}$ and $\delta=5 \mathrm{~mm}$. The figure shows that an increase in $W$ is directly linked to a decrease of the charge induced in the droplet. It implies that the hole in the bottom plate affects the geometry assumed in section 2.1 as soon as $W$ is to large. Note that the radius of the hole cannot be too small. Indeed, the droplet have to be able to leave the generator. Consequently, $W$ has to be larger than the radius of the droplet.

\subsection{Influence of $V$}

Afterwards, the influence of the voltage $V$ between the metal disks has been studied. The measurements made on water are plotted in red triangles in Fig. 5 for which the charge normalized by the surface of the droplet is reported as a function of the voltage. Fig. 5 clearly shows the linear behavior of the charge in the droplet as a function of the voltage. Measurements confirm the linear behavior stated by the Eq. (2) in the range of voltage studied (i.e. from 0 to $200 \mathrm{~V}$ ).

Finally, the variation of the polarization between both metal disks has been studied. As predicted in Eq. (2), this variation only induce a change in the sign of the charge. Indeed, when the higher voltage is on the top disk, the droplet is charged positively and when the higher voltage is on the bottom disk, the droplet is charged negatively. Moreover, we did not observe any change in the absolute value of the charge. Note that this last observation has been made for all liquids listed in the Tab.??tab1

\subsection{Best design of the charged droplet generator}

According to Eq. (2), it seems that, for a given droplet radius, $r_{d}$, the best configuration to charge the droplet is reached when $d$ is about zero and $V$ is as important as allowed by the power supply. In practice, the charge induced in the droplet begins to decrease for small $d$ compare to $\delta$. The optimal $d$ is then defined as the limit where the Eq. (2) remains valid. In our configuration $\left(r_{n}\right.$ set on 3 or $5 \mathrm{~mm}$ ), this limit is $d=5 \mathrm{~mm}$. Once $d$ has been minimized, it is also possible to increase the charge by rising V. This increase is also limited by two effects: (i)

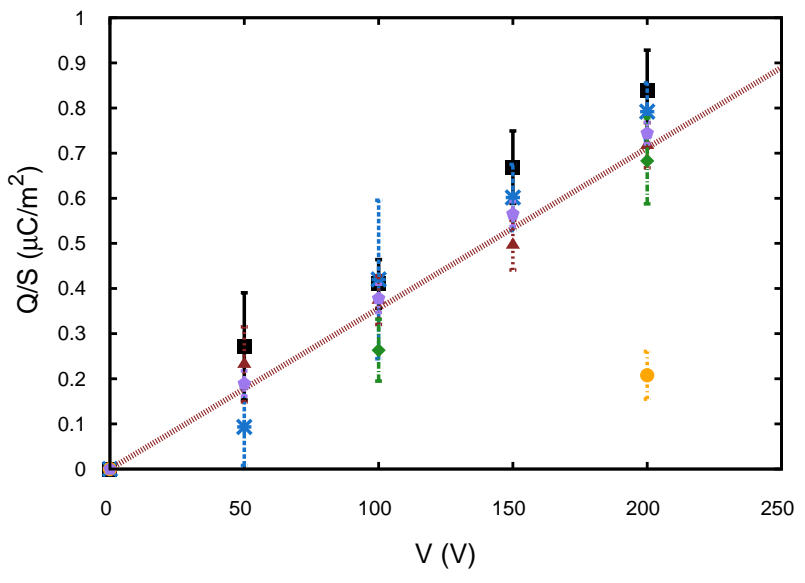

Fig. 5. (Color online) Charge induced in a droplet, normalized by the droplet surface, as a function of the voltage applied on the charged droplet generator. Red triangles, purple pentagons, Black squares, Blue stars, Green diamonds and Yellow circles represent respectively measurements made on bidistilled water, Triton- $\mathrm{x}-100$, acetone, ethanol, n-octane and silicon oil. The red continuous line is a linear fit on these measurements.

electrical breakdown may occur between the plates and (ii) for a too large voltage, a Taylor cone is formed, and droplets are ejected from the needle before reaching their maximum size. This limit corresponds to the formation of an electrospray. It is expressed as follow :

$$
Q_{R}=2 \pi \sqrt{16 \gamma \epsilon_{0} r_{d}^{3}}
$$

with $\gamma$ the surface tension of the liquid. Eq. (2), indicates also that an increase in $r_{d}$ increase the charge induced in the droplet. However, this parameter is generally fixed by experiment requirements. Finally, $W$, the radius of the hole in the bottom plate has to be reduced to maximize the charge induced in the droplet. However, it has to be large enough to allow the droplet to fall away from the droplet generator. Consequently, its value has to be calculated to be as short as possible in regard to the droplet radius. In consequence, our optimal set of parameters is $d=5 \mathrm{~mm}, \delta=2 \mathrm{~mm}$ and $W=2.5 \mathrm{~mm}$. Indeed, $\delta=2 \mathrm{~mm}$ allows to avoid any droplet wetting on the upper plate while allowing to use the planar capacitor hypothesis.

In terms of dimensionless parameters, the optimal geometry of this charged droplet generator has to follow the Eq. :

$$
w=2 r_{d} \quad \delta=2 r_{d}=\frac{d}{2} \quad v=v_{\text {Breakdown }}
$$

And, in a more general way, the set of parameters has to repsect

[7] $\frac{d-\delta}{w}>1$

which implies that $W$ and $\delta$ will be negligible from the point of view of the planar capacitor hypothesis. 


\begin{tabular}{|c|c|c|c|c|c|c|c|}
\hline & bidistilled water & $\mathrm{HCl}(1 \mathrm{M})$ & Triton X-100 & Acetone & Ethanol & n-octane & Silicone oil \\
\hline$\sigma(\mathrm{mS} / \mathrm{cm})$ & $5.510^{-5}$ & 330 & $5.510^{-5}$ & $2.110^{-4}$ & $1,110^{-6}$ & $10^{-6}$ & $10^{-11}$ \\
\hline$r_{d}(\mathrm{~mm})$ & 1.63 & 1.56 & 1.25 & 1.21 & 1.19 & 1.24 & 1.11 \\
\hline$\gamma(\mathrm{mN} / \mathrm{m})$ & 70.8 & 63.6 & 32.0 & 23.7 & 21.7 & 21.8 & 19.0 \\
\hline Polarity & polar & polar & polar & polar & polar & non-polar & non-polar \\
\hline Ions in solution & yes & yes & yes & no & yes & no & no \\
\hline$\epsilon_{r}$ & 80.1 & 4.6 & 80.1 & 20.7 & 24.3 & 2 & 2.9 \\
\hline$\tau(\mathrm{s})$ & $1.310^{-4}$ & $1.110^{-12}$ & $1.310^{-4}$ & $8.710^{-6}$ & $1.910^{-3}$ & $1.810^{-4}$ & 25.5 \\
\hline
\end{tabular}

Table 1. Properties of the considered liquids: the conductivity $(\sigma)$, the surface tension $(\gamma)$, the polarity, the presence (or not) of ions in solution of the liquids charged, the relative permittivity $\left(\epsilon_{r}\right)$ and the relaxation time $(\tau)$. All these measurements was reported at room temperature. The conductivity values are drawn from [13] and [14] while the surface tension measurements has been made by pendant droplet measurements on a CAM 200 (KSV Ltd).

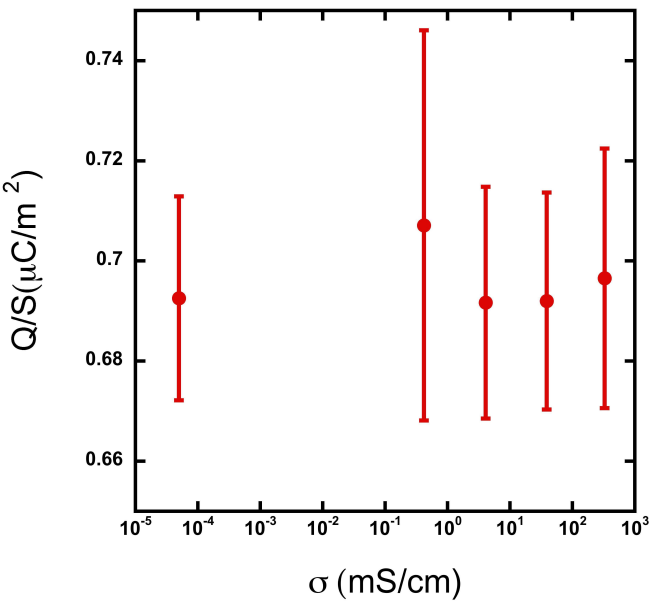

Fig. 6. (Color online) Charge induced in $\mathrm{HCl}$ droplets with different molarities, normalized by the droplet surface, as a function of the liquid conductivity. The highest conductivity correspond to a droplet of HCL $1 \mathrm{M}$.

\section{Influence of the liquid}

In order to check the domain of validity of the "perfect conductor" hypothesis, several liquids were used to obtain charged droplets. The fluids tested were : bidistilled water, a mixture of water and Triton X-100, ethanol, acetone, n-octane and silicone oil (with a viscosity of $1.5 \mathrm{cSt}$, near the water viscosity). Each of these liquids have different fluid and electrical properties. In Table 1, the conductivity $\sigma$, the surface tension $\gamma$, the polarity, the presence of ions in solution are reported and the relative permittivity are reported.

The Fig. 5 (a) shows results of measurements on the charge induced in a droplet for a voltage applied on the charged droplet generator between 0 and $200 \mathrm{~V}$. The charge of the droplet was normalized by the surface of the droplet, $4 \pi r_{d}^{2}$ (the droplet radii were measured as explained in Sect. 2.1). This normalization is explained by the fact that the different liquids do not have the same surface tension.

The first measurements (i.e. measurements which aim to study the geometry of the charged droplet generator) was made with bidistilled water and are in agreement with the "perfect conductor" hypothesis. Moreover, in the Fig. 6, we report the measurements made with solutions of $\mathrm{HCl}$ (from 0 to $1 \mathrm{M}$ ). We found that, using our device, the $\mathrm{HCl}$ mixtures does not be- have differently from water. This confirms that the water fulfilled perfect conductor conditions. Besides, bidistilled water has specifics properties. It is a polar liquid with ions in solution and a high surface tension. We extend then our study to other liquids in order to check the influence of these properties.

The liquid named Triton X-100 in the Table 1 is bidistilled water with an addition of 1CMC (critical micelle concentration, which correspond to $0.23 \mathrm{mM}$ ) of the non-ionic surfactant Triton X-100. This liquid was used to check the influence of the surface tension on the charge induced in the droplet. Acetone does not possess ions in solutions, it is then possible to check whether the charge in the droplet is due to ion (which compose the liquid) migration. Acetone and ethanol are also polar. On the contrary, n-octane does not possess ions in solution and is apolar. It is thus possible to verify the influence of the molecules polarity. Finally, silicone oil at $1.5 \mathrm{cst}$ is a liquid with viscosity near water but with a smaller electrical conductivity than any other liquids tested.

The Fig. 5 shows that almost all of the liquids tested become electrically charged in the same way. Because of the errors bars, no distinction can be made between the charge induced in the different liquids. The silicone oil is the only tested liquid which does not charge as well as the others. Indeed, it does not charge significantly below 200V. Let us remind that these observations are made between 0 and $200 \mathrm{~V}$, and thus at a small voltage compare to the electrospray limit [9]. In this configuration, it seems that it is not surfactant, ions in solution or molecules polarity which leads the droplet to charge. These observations confirm the assumption generally made which suppose that it always exist free charges in a liquid (coming from redox reactions, exchanges with gas around the droplet or impurities). As a consequence, the only relevant parameter in this system is the conductive relaxation time of the liquid used. Our measurements allow to determine the limit of electrical conductivity (with respect to the relative permittivity) below which the "perfect conductor" hypothesis is valid. For example, In the case of a liquid with a relative permittivity equal to 10 , a liquid with a conductivity less than $10^{-9} \mathrm{mS} / \mathrm{cm}$ cannot be charged using to our system.

\section{Conclusion}

In conclusion, an electrically charged droplet generator has been developed. This device was designed to generate charged droplets from any liquid. This study has also underlined how 
to optimize the different parameters of the generator. It has been proved that the perfect conductor approach is in agreement with the measurements made on the apparatus at a voltage between 0 and $200 \mathrm{~V}$. Moreover, a detailed study was performed on the influence of the liquid on the charge induced in the droplet. It results from this study that a large panel of liquids could be charged by the generator. We found that any fluid with a conductive relaxation time smaller than 1s charges like a perfect conducting fluid. Our device allows then to study the influence of an electrical charge on fundamental properties of the fluid like the surface tension or the wettability on a surface.

\section{Acknowledgements}

MB is financed by Micromast AIP (P7/38) BELSPO, Belgium. SD is an F.R.S.-FNRS research associate. This study was financially supported by the ESA topical team Poladrop and "Fonds Spécifiaux de la Recherche" (ULg).

\section{References}

1. C. Saunders, Space Sci. Rev. 137, 335 (2008).

2. L. Rayleigh, Philos. Mag. 14, 184 (1882).

3. S. J. Gaskell, J. Mass Spectrom. 32, 677 (1997).

4. F. Mugele, and J. C. Baret, J. Phys. : Condens. Matter 17, 705 (2005).

5. J. H. Wendorff, S. Agarwal, A. Greiner, Wiley-VCH (2012).

6. B. S. Hamlin, J. C. Creasey, and W. D. Ristenpart, Phys. Rev. Lett. 109, 094501 (2012).

7. R. A. Millikan, Phys. Rev. 2, 109 (1913).

8. A. R. Jones and K. C. Thong, J. Phys. D : Appl. Phys. 4, 1159 (1971).

9. D. B. Hibbert, MacMillan (1993).

10. E. Giglio, B. Gervais, J. Rangam, B. Manil, B.A. Huber, D. Duft, R. Muller, T. Leisner, and C. Guet, Phys. Rev. E 77, 036319 (2008).

11. L. P. Santos, T. R. D. Ducati, L. B. S. Balestrin, and Fernando Galembeck, J. Phys. chem. C 115, 11226 (2011).

12. D. Choi, H. Lee, D. J. Im, I. S. Kang, G. Lim, D. S. Kim, and K. H. Kang, Sci. Rep. 3, 2037 (2013).

13. P.N. Tshibangu, S. N. Ndwandwe, and E. Dixon Dikio, Int. J. Electrochem. Sci. 6, 2201 (2011).

14. H. Moretto, M. Schulze, and G. Wagner, Ullmann's encycplopedia of industrial chemistry (Weinheim, 2012). 